\title{
4-二甲氨基黄酮衍生物的合成及其活性评价
}

\author{
苏亚涁＼cjkstart洪 琛 李 骞 罗 稳* 王超杰* \\ (河南大学 天然药物与免疫工程重点实验室 开封 475004)
}

\begin{abstract}
摘要 设计合成了 10 个 4-二甲氨基黄酮衍生物, 产物结构均经 ${ }^{1} \mathrm{H}$ NMR, ESI-MS 和元素分析确认. 采用噻唑蓝(MTT) 法测试了化合物对 HepG2(人肝癌细胞)的抑制作用，结果表明目标化合物在 $30 \mu \mathrm{mol} / \mathrm{L}$ 浓度下对 HepG2 细胞损伤均有 一定的抑制作用，大部分化合物优于对照药物䚞皮素，其中化合物 $\mathbf{5 c}, \mathbf{5 e}, \mathbf{5 f}$ 和 $\mathbf{5 j}$ 活性最强，抑制率分别为 $91.0 \%$, 90.1\%, 95.7\%和 92.1\%, 而且化合物 $\mathbf{5 f}$ 在 $10 \mu \mathrm{mol} / \mathrm{L}$ 浓度下对 HepG2 的抑制率为 $93.1 \%$, 具有深入研究的价值.
\end{abstract}

关键词 黄酮; 衍生物; 合成; 抗肿瘤

\section{Synthesis and Biological Evaluation of 4-Dimethylamino Flavonoid Derivatives}

\author{
$\mathrm{Su}$, Yabin \\ Hong, Chen \\ Li, Qian \\ Luo, Wen* \\ Wang, Chaojie* \\ (Key Laboratory of Natural Medicine and Immuno-Engineering of Henan Province, Henan University, Kaifeng 475004)
}

\begin{abstract}
Ten novel 4-dimethylamino flavonoid derivatives were designed and synthesized. The structures of these compounds were confirmed by ${ }^{1} \mathrm{H}$ NMR, ESI-MS and elemental analysis. Their preliminary antitumor activity was evaluated by measuring their cytotoxic activity against the HepG2 cell line using thiazolyl blue tetrazolium bromide (MTT) assay. All ten compounds exhibited potent antitumor activity at $30 \mu \mathrm{mol} / \mathrm{L}$. Some of the compounds displayed higher activity than the reference compound, quercetin. Compounds $\mathbf{5 c}, \mathbf{5 e}, \mathbf{5 f}$ and $\mathbf{5 j}$ were the most potent with inhibition rates of 91.0\%, 90.1\%, 95.7\% and $92.1 \%$, respectively. Moreover, compound $\mathbf{5 f}$ at $10 \mu \mathrm{mol} / \mathrm{L}$ showed an inhibition rate of $93.1 \%$. Further investigation is needed.
\end{abstract}

Keywords flavonoid; derivative; synthesis; antitumor

黄酮(Flavonoids)是一类普遍存在于自然界的天然

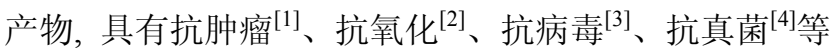
多种生理活性，而且黄酮性质温和、毒副作用较低 ${ }^{[5]}$, 在 医药领域具有广阔的应用前景. 黄酮衍生物的合成及其 生物活性方面的研究一直十分活跃, 特别是在预防慢性 疾病和发病机制复杂的疾病, 如肿瘤、阿尔茨海默症等 方面引起了人们广泛关注 ${ }^{[6]}$. 然而天然黄酮常常存在活 性不够强、水溶性不好、生物利用度差等缺点, 因此对 其结构改造和修饰一直是药物化学领域的研究热点.

我们实验室长期以来一直从事抗肿瘤药物的研 究 ${ }^{[7,8]}$, 而且前期报道了一些具有较强抗肿瘤作用的黄 酮类衍生物 ${ }^{[9,10]}$. 近来, 我们在进行体外抗肿瘤活性篮 选时, 发现 4-二甲氨基黄酮衍生物具有较好的抑制肿瘤
细胞增值活性，而且此类黄酮衍生物具有较好的水溶 性. 因此本文报道了 10 个新的 4-二甲氨基黄酩衍生物 的合成及其初步的抗肿瘤活性评价，希望对今后黄酮类 抗肿瘤药物的设计提供依据. 其合成路线见图 1 .

\section{1 结果与讨论}

\section{1 合成与结构}

我们曾尝试查尔酮路线合成黄酮母核 3, 以 2,4-二 羟基苯乙酮为起始原料, 在碱性条件下与芳香醛缩 合 ${ }^{[1]}$, 然而由于酚羟基的存在，收率很低，小于 $10 \%$. 我们也曾参考文献[12]方法，以硼酸为催化剂合成查尔 酮, 产率有所提高, 为 $41 \%$, 但是蒸干溶剂后为红色粘 稠固体, 萃取时乳化严重, 而且需要柱层析分离. 所得

\footnotetext{
*E-mail: luowen83@hehu.edu.cn

Received September 26, 2013; revised October 29, 2013; published online November 5, 2013.

Project supported by the National Natural Science Foundation of China (Nos. 21172053, 21302041), the Postdoctoral Science Foundation of China (No. 2012M521391) and the Postdoctoral Science Foundation of Henan Province (No. BH2011043).

国家自然科学基金(Nos. 21172053, 21302041)、中国博士后基金(No. 2012M521391)、河南省博士后基金(No. BH2011043)资助项目.
} 


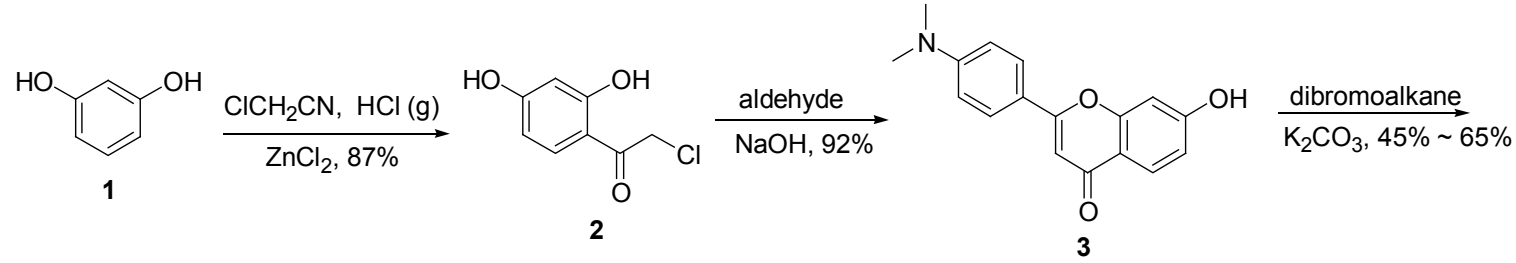

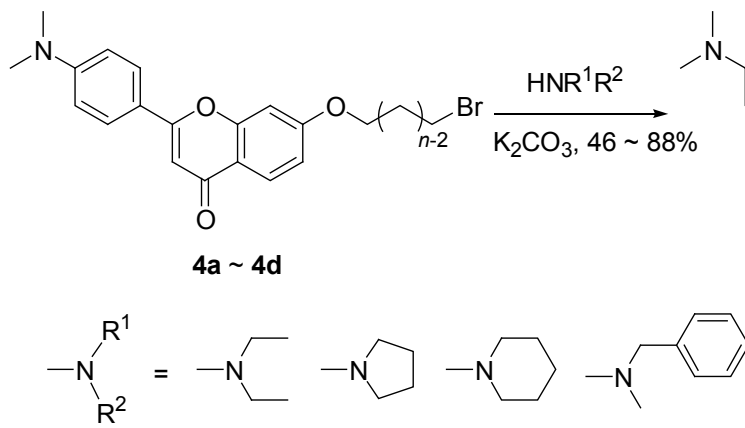

图 1 黄酮衍生物的合成路线

Figure 1 Synthesis of flavonoid derivatives

到的查尔酮在碘和浓硫酸的催化下关环, 没有得到预期 产物 3. 因此我们通过文献调研 ${ }^{[13]}$, 选择本文采用的路 线进行黄酮母核 3 的合成, 产率较高, 处理方便.

图 1 路线是以廉价的间苯二酚 $\mathbf{1}$ 为起始原料, 在氯 化锌、盐酸气体作用下与氯乙腈反应, 后水解生成化合 物 2 , 产率 $87 \%$, 纯度较高, 水重结晶后可直接进行下 一步反应. 化合物 2 在乙醇中, 稀氢氧化钠存在下, 与 4-二甲氨基苯甲醛反应, 直接生成黄酮母核 3, 产率为 $92 \%$, 乙醇重结晶，直接用于下一步反应. 黄酮母核 3 在丙酮中. 无水碳酸钾的作用下与过量的二溴烷烃作 用, 生成中间体 $\mathbf{4 a} \sim \mathbf{4 d}$, 柱层析产品损失较多, 因此采 用乙醇重结晶方法提纯. 目标化合物 $\mathbf{5 a} \sim 5 \mathbf{j}$ 的合成是 将中间体 $\mathbf{4 a \sim 4 d}$ 加入到乙腈中, 在无水碳酸钾和不同 的仲胺反应生成, 产率 $46 \% \sim 88 \%$, 用氯仿/甲醇/氨水 柱层析可得到纯品, 部分化合物需要再用乙醇重结晶.

由 ${ }^{1} \mathrm{H}$ NMR 和 ${ }^{13} \mathrm{C}$ NMR 数据可以确定化合物的结 构, 元素分析结果显示 $\mathrm{C}, \mathrm{H}, \mathrm{N}$ 元素含量均在误差范围 内. 质谱分子离子峰也显示出与预期相符的结果.

\section{2 生理活性测试}

采用噻唑蓝(MTT)法 ${ }^{[14]}$ 测试了化合物 $\mathbf{5 a} \sim \mathbf{5} \mathbf{j}$ 对人 肝癌细胞 $\mathrm{HepG} 2$ 的体外抑制率, 测试浓度为 10 和 30 $\mu \mathrm{mol} / \mathrm{L}$, 檞皮素作为对照药物, 结果如表 1 所示. 由表 可以看出, 化合物 $\mathbf{5 a} \sim \mathbf{5 j}$ 和檞皮素在测试浓度下对 HepG2 细胞均有不同程度的抑制作用, 且呈浓度依赖 性; 大部分化合物的活性高于对照药物槲皮素, 其中化 合物 $\mathbf{5 c}, \mathbf{5 e}, \mathbf{5 f}$ 和 $\mathbf{5 j}$ 活性最好, $30 \mu \mathrm{mol} / \mathrm{L}$ 浓度下损伤抑 制率分别为 $91.0 \%, 90.1 \%, 95.7 \%$ 和 $92.1 \%$, 化合物 $\mathbf{5 f}$ 在 $10 \mu \mathrm{mol} / \mathrm{L}$ 浓度下抑制率仍为 $93.1 \%$, 具有深入研究的 价值.
表 1 化合物 $\mathbf{5 a} \sim \mathbf{5 j}$ 对 HepG2 细胞抑制率

Table 1 The inhibition activity of compounds $\mathbf{5 a} \sim \mathbf{5} \mathbf{j}$ for HepG2 cell lines in vitro

\begin{tabular}{|c|c|c|c|c|}
\hline \multirow{2}{*}{ Compd. } & \multirow{2}{*}{$n$} & \multirow{2}{*}{$\mathrm{NR}^{1} \mathrm{R}^{2}$} & \multicolumn{2}{|c|}{ 抑制率 ${ }^{a}$} \\
\hline & & & $10 \mu \mathrm{mol} / \mathrm{L}$ & $30 \mu \mathrm{mol} / \mathrm{L}$ \\
\hline $5 a$ & 4 & & $19.9 \pm 4.1$ & $77.3 \pm 3.8$ \\
\hline $5 b$ & 4 & & $15.6 \pm 6.2$ & $76.5 \pm 9.4$ \\
\hline $5 c$ & 6 & & $41.2 \pm 8.3$ & $91.0 \pm 3.8$ \\
\hline $5 d$ & 6 & & $0.6 \pm 0.5$ & $35.0 \pm 3.8$ \\
\hline $5 e$ & 8 & & $51.7 \pm 9.7$ & $90.1 \pm 2.7$ \\
\hline $5 f$ & 8 & & $93.1 \pm 1.3$ & $95.7 \pm 0.5$ \\
\hline $5 \mathrm{~g}$ & 8 & & $14.9 \pm 1.2$ & $26.2 \pm 1.0$ \\
\hline $5 \mathrm{~h}$ & 8 & & $2.8 \pm 0.4$ & $7.8 \pm 1.1$ \\
\hline $5 i$ & 10 & & $7.0 \pm 0.8$ & $18.5 \pm 4.9$ \\
\hline $5 \mathbf{j}$ & 10 & $-N$ & $17.3 \pm 2.9$ & $92.1 \pm 1.6$ \\
\hline Quercetin & - & 一 & $16.5 \pm 1.2$ & $33.2 \pm 2.8$ \\
\hline
\end{tabular}

比较化合物的结构与活性, 可以发现, 碳链的长度 
对化合物的活性有影响, 链中碳的个数在 8 的时候活性 最好, 如化合物 5e 和 5f, 太长或太短均使活性降低. 长 链末端的取代基对活性也有影响, 碳链相同时, 二乙胺 和吡咯烷取代的化合物活性优于哌啶和 $N$-甲基苠胺取 代的化合物.

\section{2 实验部分}

\section{1 仪器与试剂}

Bruker AV-400 型核磁共振仪(美国); Esquire3000 型 LC-MS 质谱仪(德国); X-6 型显微熔点仪(中国, 温度计 未校正); VarioEL III 型元素分析仪(德国); WFH-204B型 手提式紫外灯; BIO-TEKELX-800 型酶标仪(美国); XD-101 型倒置生物显微镜(中国); H-B11600 型电热恒 温培养箱(中国).

间苯二酚、氯乙腈、4-二甲氨基苯甲醛(上海阿拉丁 试剂有限公司), 槲皮素(中国药品生物制品检定所), 硅 胶(青岛海浪硅胶厂, 200 300目, HepG2 细胞(中国科学 院上海细胞库), 其他试剂均为市售分析纯, 未做进一 步处理.

\section{2 合成部分}

\subsubsection{2-氯-1-(2,4-二羟基苯基)乙酮(2)的合成}

将间苯二酚(11.3 g, 0.1 mol)、氯乙腈 $(15.1 \mathrm{~g}, 0.2 \mathrm{~mol})$ 与无水氯化锌 $(5.4 \mathrm{~g}, 0.4 \mathrm{~mol})$ 混合, 加入无水乙醚 50 $\mathrm{mL}$, 用冰盐浴冷却, 通入干燥的氯化氢气体, $2 \mathrm{~h}$ 后将反 应瓶于冰箱中放置 $1 \mathrm{~d}$, 再通氯化氢 $2 \mathrm{~h}$, 冰箱中放置 $3 \mathrm{~d}$, 倾出乙醚, 用热水把粗提物转到圆底烧瓶中, 回流 $1 \mathrm{~h}$, 静置过夜, 过滤, 滤饼用水重结晶, 得化合物 3 (16.2, $87 \%)$.

\subsubsection{4-二甲氨基黄酮(3)的合成}

将化合物 2 (1.9 g, $10.0 \mathrm{mmol})$ 与 4-二甲氨基苯甲醛 $(1.5 \mathrm{~g}, 20.0 \mathrm{mmol})$ 混合, 加入 $95 \%$ 乙醇 $40 \mathrm{~mL}$, 搅拌下滴 加 $10 \% \mathrm{NaOH}(15 \mathrm{~mL})$, 室温下反应 $2 \mathrm{~d}$, 反应完毕, 用 $10 \%$ 盐酸调 $\mathrm{pH}=7$, 再于室温下搅拌 $6 \mathrm{~h}$, 抽滤, 滤饼用 95\%乙醇重结晶，得黄酮母核 3 (2.5 g, 92\%). ${ }^{1} \mathrm{H}$ NMR $\left(400 \mathrm{MHz}, \mathrm{DMSO}-d_{6}\right) \delta: 7.80(\mathrm{~d}, J=9.0 \mathrm{~Hz}, 2 \mathrm{H}), 7.58$ (d, $J=8.4 \mathrm{~Hz}, 1 \mathrm{H}), 6.80$ (d, $J=9.0 \mathrm{~Hz}, 2 \mathrm{H}), 6.78$ (d, $J=1.9$ $\mathrm{Hz}, 1 \mathrm{H}), 6.73$ (s, 1H), $6.70(\mathrm{dd}, J=8.4,1.9 \mathrm{~Hz}, 1 \mathrm{H}), 3.02$ (s, 6H); MS (ESI) $m / z: 282.1(\mathrm{M}+\mathrm{H})^{+}$.

\subsection{3中间体 $4 \mathrm{a} \sim 4 \mathrm{e}$ 的合成通法}

将化合物 3 (0.56 g, $2.0 \mathrm{mmol})$, 无水 $\mathrm{K}_{2} \mathrm{CO}_{3}(1.38 \mathrm{~g}$, $10.0 \mathrm{mmol})$ ，二溴烷烃 $(6.0 \mathrm{mmol})$ 加入到 $10 \mathrm{~mL}$ 丙酮中, 加热回流 $10 \mathrm{~h}, \mathrm{TLC}$ 检测反应至原料消失. 减压蒸除溶 剂, 加入 $30 \mathrm{~mL}$ 水, 乙酸乙酯萃取 3 次, 无水 $\mathrm{Na}_{2} \mathrm{SO}_{4}$ 干 燥后, 蒸干溶剂, 乙醇重结晶得中间体 $\mathbf{4 a} \sim \mathbf{4 e}$, 不用提
纯直接用于下一步反应.

\subsection{4 目标化合物 $\mathbf{5 a} \sim \mathbf{5} \mathbf{j}$ 的合成通法}

将 $\mathbf{4 a} \sim 4 \mathbf{e}(1.0 \mathrm{mmol})$ 溶于 $10 \mathrm{~mL}$ 乙腈中，加入无水 $\mathrm{K}_{2} \mathrm{CO}_{3}(0.69 \mathrm{~g}, 5.0 \mathrm{mmol})$ 和不同的仲胺 $(2.0 \mathrm{mmol}), 60$ ${ }^{\circ} \mathrm{C}$ 搅拌 $8 \mathrm{~h}$, 反应完毕, 减压蒸除溶剂, 加入 $30 \mathrm{~mL}$ 氯仿, 依次用水和饱和食盐水洗涤，有机层用无水 $\mathrm{Na}_{2} \mathrm{SO}_{4}$ 干 燥，蒸干溶剂，氯仿/甲醇/氨水(30：1：0.5\%)柱层析， 然后乙醇重结晶可得目标化合物 $\mathbf{5 a} \sim \mathbf{5 j}$.

7-[4-(二乙基氨基)丁氧基]-2-[4-(二甲氨基)苯 基]-4H-苯并吡喃-4-酮(5a)：产率 $61 \%$. 黄色固体，m.p. $114 \sim 116{ }^{\circ} \mathrm{C} ;{ }^{1} \mathrm{H}$ NMR (400 MHz, $\left.\mathrm{CDCl}_{3}\right) \delta: 7.83(\mathrm{~d}, J=$ $8.8 \mathrm{~Hz}, 2 \mathrm{H}), 7.70$ (d, $J=8.4 \mathrm{~Hz}, 1 \mathrm{H}), 6.84(\mathrm{~s}, 1 \mathrm{H}), 6.79 \sim$ $6.71(\mathrm{~m}, 4 \mathrm{H}), 4.10(\mathrm{t}, J=6.4 \mathrm{~Hz}, 2 \mathrm{H}), 3.07(\mathrm{~s}, 6 \mathrm{H}), 2.60 \sim$ $2.48(\mathrm{~m}, 6 \mathrm{H}), 1.86(\mathrm{dd}, J=14.5,6.9 \mathrm{~Hz}, 2 \mathrm{H}), 1.83 \sim 1.64$ $(\mathrm{m}, 2 \mathrm{H}), 1.05(\mathrm{t}, J=7.1 \mathrm{~Hz}, 6 \mathrm{H}) ;{ }^{13} \mathrm{C}$ NMR $(100 \mathrm{MHz}$, $\left.\mathrm{CDCl}_{3}\right) \delta: 182.60,167.69,166.24,151.09,145.90,133.28$, $125.39,120.11,115.42,113.87,112.13,111.90,96.85$, 68.64, 58.33, 52.46, 46.81, 40.11, 27.08, 23.53, 18.48, 11.67; $\mathrm{MS}\left(\mathrm{ESI}^{+}\right) \mathrm{m} / \mathrm{z}: 409.1[\mathrm{M}+\mathrm{H}]^{+}$. Anal. calcd for $\mathrm{C}_{25} \mathrm{H}_{32} \mathrm{~N}_{2} \mathrm{O}_{3}$ : C 73.50, $\mathrm{H}$ 7.90, $\mathrm{N}$ 6.86; found $\mathrm{C} 73.54, \mathrm{H}$ 7.90, N 6.76 .

7-[4-(吡咯烷-1-基)丁氧基]-2-[4-(二甲基氨基)苯 基]-4H-苯并吡喃-4-酮(5b): 产率 56\%. 黄色固体, m.p. $124 \sim 126{ }^{\circ} \mathrm{C} ;{ }^{1} \mathrm{H}$ NMR $\left(400 \mathrm{MHz}, \mathrm{CDCl}_{3}\right) \delta: 7.82(\mathrm{~d}, J=$ $8.8 \mathrm{~Hz}, 2 \mathrm{H}), 7.70$ (d, $J=8.5 \mathrm{~Hz}, 1 \mathrm{H}), 6.84(\mathrm{~s}, 1 \mathrm{H}), 6.79 \sim$ $6.69(\mathrm{~m}, 4 \mathrm{H}), 4.10(\mathrm{t}, J=6.4 \mathrm{~Hz}, 2 \mathrm{H}), 3.07(\mathrm{~s}, 6 \mathrm{H}), 2.53(\mathrm{t}$, $J=7.1 \mathrm{~Hz}, 6 \mathrm{H}), 1.91 \sim 1.70(\mathrm{~m}, 8 \mathrm{H}) ;{ }^{13} \mathrm{C} \mathrm{NMR}(100 \mathrm{MHz}$, $\left.\mathrm{CDCl}_{3}\right) \delta: 182.56,167.66,166.21,151.07,145.88,133.27$, $125.34,120.08,115.41,113.84,112.13,111.88,96.84$, 68.55, 56.02, 54.23, 40.09, 27.09, 25.47, 23.42; $\mathrm{MS}\left(\mathrm{ESI}^{+}\right)$ $m / z: 407.1[\mathrm{M}+\mathrm{H}]^{+}$. Anal. calcd for $\mathrm{C}_{25} \mathrm{H}_{30} \mathrm{~N}_{2} \mathrm{O}_{3}$ • $0.4 \mathrm{C}_{2} \mathrm{H}_{5} \mathrm{OH} \bullet 0.1 \mathrm{CHCl}_{3}$ : C 71.20, $\mathrm{H} \mathrm{7.50,} \mathrm{N} \mathrm{6.41;} \mathrm{found} \mathrm{C}$ 71.46, H 7.23, N 6.44.

7-[6-(二乙基氨基) 己氧基]-2-[4-(二甲氨基) 苯 基]-4H-苯并吡喃-4-酩(5c)：产率 $60 \%$. 黄色固体，m.p. $109 \sim 112{ }^{\circ} \mathrm{C} ;{ }^{1} \mathrm{H}$ NMR $\left(400 \mathrm{MHz}, \mathrm{CDCl}_{3}\right) \delta: 7.82(\mathrm{~d}, J=$ $9.0 \mathrm{~Hz}, 2 \mathrm{H}), 7.70$ (d, $J=8.4 \mathrm{~Hz}, 1 \mathrm{H}), 6.84(\mathrm{~s}, 1 \mathrm{H}), 6.78 \sim$ $6.69(\mathrm{~m}, 4 \mathrm{H}), 4.07$ (t, $J=6.5 \mathrm{~Hz}, 2 \mathrm{H}), 3.07$ (s, 6H), 2.54 (q, $J=7.2 \mathrm{~Hz}, 4 \mathrm{H}), 2.48 \sim 2.40(\mathrm{~m}, 2 \mathrm{H}), 1.87 \sim 1.82(\mathrm{~m}, 2 \mathrm{H})$, $1.56 \sim 1.47(\mathrm{~m}, 4 \mathrm{H}), 1.42 \sim 1.36(\mathrm{~m}, 2 \mathrm{H}), 1.04(\mathrm{t}, J=7.2$ $\mathrm{Hz}, 6 \mathrm{H}) ;{ }^{13} \mathrm{C} \mathrm{NMR}\left(100 \mathrm{MHz}, \mathrm{CDCl}_{3}\right) \delta: 182.56,167.68$, $166.28,151.07,145.90,133.26,125.35,120.12,115.40$, $113.80,112.13,111.89,96.83,68.74,52.85,46.90,40.10$, 28.98, 27.43, 26.99, 26.00, 11.69; $\mathrm{MS}\left(\mathrm{ESI}^{+}\right) \mathrm{m} / \mathrm{z}: 437.3$ $[\mathrm{M}+\mathrm{H}]^{+}$. Anal. calcd for $\mathrm{C}_{27} \mathrm{H}_{36} \mathrm{~N}_{2} \mathrm{O}_{3}$ : C 74.28, $\mathrm{H}$ 8.31, N 
6.42; found C 74.53, H 8.33, N 6.41.

7-[6-(吡咯烷-1-基)己氧基]-2-[4-(二甲基氨基)苯 基]-4H-苯并吡喃-4-酮(5d): 产率 88\%. 黄色固体, m.p. $139 \sim 141{ }^{\circ} \mathrm{C} ;{ }^{1} \mathrm{H}$ NMR $\left(400 \mathrm{MHz}, \mathrm{CDCl}_{3}\right) \delta: 7.75$ (d, $J=$ $8.9 \mathrm{~Hz}, 2 \mathrm{H}), 7.64$ (d, $J=9.0 \mathrm{~Hz}, 1 \mathrm{H}), 6.77$ (s, 1H), 6.67 (d, $J=9.3 \mathrm{~Hz}, 4 \mathrm{H}), 3.99$ (t, $J=6.5 \mathrm{~Hz}, 2 \mathrm{H}), 2.99$ (s, 6H), 2.48 $(\mathrm{d}, J=6.4 \mathrm{~Hz}, 4 \mathrm{H}), 2.46 \sim 2.39(\mathrm{~m}, 2 \mathrm{H}), 1.86 \sim 1.67(\mathrm{~m}$, $6 \mathrm{H}), 1.56 \sim 1.50(\mathrm{~m}, 2 \mathrm{H}), 1.48 \sim 1.42(\mathrm{~m}, 2 \mathrm{H}), 1.41 \sim 1.31$ $(\mathrm{m}, 2 \mathrm{H}) ;{ }^{13} \mathrm{C} \mathrm{NMR}\left(100 \mathrm{MHz}, \mathrm{CDCl}_{3}\right) \delta: 182.47,167.62$, $166.24,151.03,145.83,133.24,125.26,120.02,115.34$, 113.82, 112.12, 111.84, 96.78, 68.70, 56.51, 54.23, 40.04, 28.91, 27.40, 25.94, 23.37; $\mathrm{MS}\left(\mathrm{ESI}^{+}\right) \mathrm{m} / z: 435.2[\mathrm{M}+$ $\mathrm{H}]^{+}$. Anal. calcd for $\mathrm{C}_{27} \mathrm{H}_{34} \mathrm{~N}_{2} \mathrm{O}_{3} \bullet 0.4 \mathrm{C}_{2} \mathrm{H}_{5} \mathrm{OH} \bullet 0.1 \mathrm{CHCl}_{3}$ : C 72.07, H 7.91, N 6.03; found C 72.55, H 7.70, N 6.25.

2-[4-(二甲氨基) 苯基]-7-[8-(二乙基氨基) 辛氧 基]-4H-苯并吡喃-4-酮(5e): 产率 $65 \%$. 黄色固体, m.p. $100 \sim 102{ }^{\circ} \mathrm{C} ;{ }^{1} \mathrm{H}$ NMR (400 MHz, $\left.\mathrm{CDCl}_{3}\right) \delta: 7.83$ (d, $J=$ $8.9 \mathrm{~Hz}, 2 \mathrm{H}), 7.70$ (d, $J=8.4 \mathrm{~Hz}, 1 \mathrm{H}), 6.84(\mathrm{~s}, 1 \mathrm{H}), 6.77 \sim$ $6.70(\mathrm{~m}, 4 \mathrm{H}), 4.07$ (t, $J=6.5 \mathrm{~Hz}, 2 \mathrm{H}), 3.07$ (s, 6H), 2.57 (q, $J=7.1 \mathrm{~Hz}, 4 \mathrm{H}), 2.49 \sim 2.42(\mathrm{~m}, 2 \mathrm{H}), 1.89 \sim 1.80(\mathrm{~m}, 2 \mathrm{H})$, $1.48(\mathrm{~d}, J=7.1 \mathrm{~Hz}, 4 \mathrm{H}), 1.37 \sim 1.31(\mathrm{~m}, 8 \mathrm{H}), 1.05(\mathrm{t}, J=$ $7.2 \mathrm{~Hz}, 6 \mathrm{H}) ;{ }^{13} \mathrm{C} \mathrm{NMR}\left(100 \mathrm{MHz}, \mathrm{CDCl}_{3}\right) \delta: 182.58$, $167.71,166.32,151.09,145.92,133.26,125.37,120.16$, $115.40,113.79,112.13,111.91,96.84,68.81,52.94,46.86$, 40.10, 29.52, 29.30, 28.97, 27.63, 26.89, 25.93, 11.57; MS $\left(\mathrm{ESI}^{+}\right) \mathrm{m} / z: 465.3[\mathrm{M}+\mathrm{H}]^{+}$. Anal. calcd for $\mathrm{C}_{29} \mathrm{H}_{40} \mathrm{~N}_{2} \mathrm{O}_{3} \bullet$ $0.4 \mathrm{C}_{2} \mathrm{H}_{5} \mathrm{OH}$ : C 74.09, H 8.85, N 5.80; found $\mathrm{C} 74.02, \mathrm{H}$ $8.68, \mathrm{~N} 5.81$.

2-[4-(二甲基氨基)苯基]-7-[8-(吡咯烷-1-基)辛氧 基]-4H-苯并吡喃-4-酮(5f): 产率 61\%. 黄色固体, m.p. $89 \sim 91{ }^{\circ} \mathrm{C} ;{ }^{1} \mathrm{H}$ NMR (400 MHz, $\left.\mathrm{CDCl}_{3}\right) \delta: 7.80(\mathrm{~d}, J=8.9$ $\mathrm{Hz}, 2 \mathrm{H}), 7.67$ (d, $J=8.5 \mathrm{~Hz}, 1 \mathrm{H}), 6.81(\mathrm{~s}, 1 \mathrm{H}), 6.76 \sim 6.65$ (m, 4H), $4.04(\mathrm{t}, J=6.5 \mathrm{~Hz}, 2 \mathrm{H}), 3.04(\mathrm{~s}, 6 \mathrm{H}), 2.51(\mathrm{~s}, 4 \mathrm{H})$, $2.46 \sim 2.37(\mathrm{~m}, 2 \mathrm{H}), 1.87 \sim 1.74(\mathrm{~m}, 6 \mathrm{H}), 1.57 \sim 1.43(\mathrm{~m}$, $4 \mathrm{H}), 1.35(\mathrm{~s}, 6 \mathrm{H}) ;{ }^{13} \mathrm{C} \mathrm{NMR}\left(100 \mathrm{MHz}, \mathrm{CDCl}_{3}\right) \delta: 182.53$, $167.68,166.31,151.08,145.90,133.25,125.33,120.14$, $115.39,113.77,112.12,111.90,96.84,68.80,56.67,54.24$, 40.07, 29.48, 29.24, 29.01, 28.96, 27.64, 25.91, 23.40; MS $\left(\mathrm{ESI}^{+}\right) \mathrm{m} / z: 463.3[\mathrm{M}+\mathrm{H}]^{+}$. Anal. calcd for $\mathrm{C}_{29} \mathrm{H}_{38} \mathrm{~N}_{2} \mathrm{O}_{3}: \mathrm{C}$ 75.29, H 8.28, N 6.06; found C 74.98, H 8.28, N 5.93.

2-[4-(二甲基氨基) 苯基]-7-[8-(哌啶-1-基) 辛氧 基]-4H-苯并吡喃-4-酮(5g): 产率 $63 \%$. 黄色固体, m.p. $130 \sim 132{ }^{\circ} \mathrm{C} ;{ }^{1} \mathrm{H}$ NMR $\left(400 \mathrm{MHz}, \mathrm{CDCl}_{3}\right) \delta: 7.83$ (d, $J=$ $8.9 \mathrm{~Hz}, 2 \mathrm{H}), 7.70$ (d, $J=8.3 \mathrm{~Hz}, 1 \mathrm{H}), 6.84(\mathrm{~s}, 1 \mathrm{H}), 6.79 \sim$ $6.68(\mathrm{~m}, 4 \mathrm{H}), 4.07(\mathrm{t}, J=6.5 \mathrm{~Hz}, 2 \mathrm{H}), 3.07(\mathrm{~s}, 6 \mathrm{H}), 2.36(\mathrm{~d}$,
$J=28.0 \mathrm{~Hz}, 4 \mathrm{H}), 2.29(\mathrm{~d}, J=7.9 \mathrm{~Hz}, 2 \mathrm{H}), 1.88 \sim 1.79(\mathrm{~m}$, $2 \mathrm{H}), 1.65 \sim 1.58(\mathrm{~m}, 4 \mathrm{H}), 1.56 \sim 1.43(\mathrm{~m}, 6 \mathrm{H}), 1.40 \sim 1.29$ $(\mathrm{m}, 6 \mathrm{H}) ;{ }^{13} \mathrm{C} \mathrm{NMR}\left(100 \mathrm{MHz}, \mathrm{CDCl}_{3}\right) \delta: 182.55,167.69$, $166.31,151.08,145.91,133.25,125.34,120.15,115.39$, $113.77,112.12,111.90,96.84,68.80,59.66,54.68,40.09$, 29.50, 29.25, 28.97, 27.68, 26.94, 26.00, 25.91, 24.51; MS $\left(\mathrm{ESI}^{+}\right) \mathrm{m} / z: 477.3[\mathrm{M}+\mathrm{H}]^{+}$. Anal. calcd for $\mathrm{C}_{30} \mathrm{H}_{40} \mathrm{~N}_{2} \mathrm{O}_{3}: \mathrm{C}$ 75.59, H 8.46, N 5.88; found C 74.97, H 8.47, N 5.76.

7-[8-(苠基(甲基)氨基)辛氧基]-2-[4-(二甲氨基)苯 基]-4H-苯并吡喃-4-酮(5h): 产率 46\%. 黄色固体, m.p. $80 \sim 82{ }^{\circ} \mathrm{C} ;{ }^{1} \mathrm{H}$ NMR $\left(400 \mathrm{MHz}, \mathrm{CDCl}_{3}\right) \delta: 7.81$ (d, $J=8.8$ $\mathrm{Hz}, 2 \mathrm{H}), 7.68$ (d, $J=8.4 \mathrm{~Hz}, 1 \mathrm{H}), 7.31$ (d, $J=4.4 \mathrm{~Hz}, 4 \mathrm{H})$, $7.24(\mathrm{~d}, J=4.3 \mathrm{~Hz}, 1 \mathrm{H}), 6.83(\mathrm{~s}, 1 \mathrm{H}), 6.76 \sim 6.71(\mathrm{~m}, 4 \mathrm{H})$, $4.05(\mathrm{t}, J=6.5 \mathrm{~Hz}, 2 \mathrm{H}), 3.48(\mathrm{~s}, 2 \mathrm{H}), 3.05(\mathrm{~s}, 6 \mathrm{H}), 2.45 \sim$ $2.29(\mathrm{~m}, 2 \mathrm{H}), 2.18(\mathrm{~s}, 3 \mathrm{H}), 1.87 \sim 1.78(\mathrm{~m}, 2 \mathrm{H}), 1.51 \sim$ $1.47(\mathrm{~m}, 4 \mathrm{H}), 1.34(\mathrm{~s}, 6 \mathrm{H}) ;{ }^{13} \mathrm{C} \mathrm{NMR}\left(100 \mathrm{MHz}, \mathrm{CDCl}_{3}\right) \delta$ : $182.57,167.71,166.33,151.10,145.93,133.27,129.11$, $128.21,126.93,125.37,120.16,115.41,113.79,112.14$, 111.92, 96.86, 68.82, 62.31, 57.50, 42.26, 40.10, 29.47, 29.31, 28.98, 27.35, 25.93; $\mathrm{MS}\left(\mathrm{ESI}^{+}\right) \mathrm{m} / z$ : $513.4[\mathrm{M}+$ $\mathrm{H}]^{+}$. Anal. calcd for $\mathrm{C}_{33} \mathrm{H}_{40} \mathrm{~N}_{2} \mathrm{O}_{3} \bullet 0.4 \mathrm{C}_{2} \mathrm{H}_{5} \mathrm{OH}: \mathrm{C} 76.44, \mathrm{H}$ 8.05, N 5.27; found C 76.46, H 7.95, N 5.27.

7-[10-(二乙基氨基) 癸氧基]-2-[4-(二甲氨基)苯 基]-4H-色烯-4-酮(5i)：产率 58\%. 黄色固体, m.p. 101 $102{ }^{\circ} \mathrm{C} ;{ }^{1} \mathrm{H}$ NMR (400 MHz, $\left.\mathrm{CDCl}_{3}\right) \delta: 7.91 \sim 7.76(\mathrm{~m}$, $2 \mathrm{H}), 7.76 \sim 7.62(\mathrm{~m}, 1 \mathrm{H}), 6.83(\mathrm{~s}, 1 \mathrm{H}), 6.81 \sim 6.68(\mathrm{~m}$, 4H), 4.06 (t, $J=6.6 \mathrm{~Hz}, 2 \mathrm{H}), 3.06(\mathrm{~s}, 6 \mathrm{H}), 2.53$ (q, $J=7.2$ $\mathrm{Hz}, 4 \mathrm{H}), 2.43 \sim 2.39(\mathrm{~m}, 2 \mathrm{H}), 1.92 \sim 1.75(\mathrm{~m}, 2 \mathrm{H}), 1.46 \sim$ $1.42(\mathrm{~m}, 4 \mathrm{H}), 1.40 \sim 1.26(\mathrm{~m}, 10 \mathrm{H}), 1.03(\mathrm{t}, J=7.2 \mathrm{~Hz}$, $6 \mathrm{H}) ;{ }^{13} \mathrm{C} \mathrm{NMR}\left(100 \mathrm{MHz}, \mathrm{CDCl}_{3}\right) \delta: 182.56,167.71$, $166.33,151.09,145.93,133.25,125.36,120.18,115.41$, 113.74, 112.12, 111.91, 96.85, 68.83, 53.03, 46.90, 40.09, $29.63,29.55,29.51,29.33,28.99,27.73,27.03,25.96$, 11.68; $\mathrm{MS}\left(\mathrm{ESI}^{+}\right) \mathrm{m} / \mathrm{z}: 493.4[\mathrm{M}+\mathrm{H}]^{+}$. Anal. calcd for $\mathrm{C}_{31} \mathrm{H}_{44} \mathrm{~N}_{2} \mathrm{O}_{3}$ : C 75.57, $\mathrm{H}$ 9.00, N 5.69; found $\mathrm{C} 75.25, \mathrm{H}$ $9.03, \mathrm{~N} 5.54$.

7-[10-(吡咯烷-1-基)癸氧基]2-[4-(二甲基氨基)苯 基]-4H-色烯-4-酮 $(\mathbf{5 j})$ ：产率 47\%. 黄色固体, m.p. 98 $100{ }^{\circ} \mathrm{C} ;{ }^{1} \mathrm{H}$ NMR (400 MHz, $\left.\mathrm{CDCl}_{3}\right) \delta: 7.81$ (d, $J=8.9$ $\mathrm{Hz}, 2 \mathrm{H}), 7.68$ (d, $J=8.3 \mathrm{~Hz}, 1 \mathrm{H}), 6.82(\mathrm{~s}, 1 \mathrm{H}), 6.76 \sim 6.69$ $(\mathrm{m}, 4 \mathrm{H}), 4.05(\mathrm{t}, J=6.5 \mathrm{~Hz}, 2 \mathrm{H}), 3.05(\mathrm{~s}, 6 \mathrm{H}), 2.54(\mathrm{~s}, 4 \mathrm{H})$, $2.48 \sim 2.42(\mathrm{~m}, 2 \mathrm{H}), 1.87 \sim 1.75(\mathrm{~m}, 6 \mathrm{H}), 1.58 \sim 1.43(\mathrm{~m}$, $4 \mathrm{H}), 1.35 \sim 1.31(\mathrm{~m}, 10 \mathrm{H}) ;{ }^{13} \mathrm{C}$ NMR $\left(100 \mathrm{MHz}, \mathrm{CDCl}_{3}\right) \delta$ : $182.58,167.70,166.32,151.07,145.90,133.26,125.35$, $120.12,115.37,113.81,112.15,111.89,96.82,68.82$, 
$56.70,54.24,40.10,29.57,29.50,29.32,28.97,28.93$, 27.69, 25.95, 23.38; $\left.\mathrm{MS}_{\left(\mathrm{ESI}^{+}\right)}\right) \mathrm{m} / z: 491.3[\mathrm{M}+\mathrm{H}]^{+}$. Anal. calcd for $\mathrm{C}_{31} \mathrm{H}_{42} \mathrm{~N}_{2} \mathrm{O}_{3} \cdot 0.5 \mathrm{C}_{2} \mathrm{H}_{5} \mathrm{OH}: \mathrm{C} 74.82, \mathrm{H} 8.83, \mathrm{~N}$ 5.45; found C 74.88, H 8.57, N 5.71.

\subsection{4 体外抗肿瘤活性测试}

使用 MTT 染色法对合成的黄酮衍生物对 HepG2(人 肝癌细胞) 进行了体外抗肿瘤活性的初步篮选, 以槲皮 素为阳性对照药物. 取对数生长周期的肿瘤细胞, 调整 细胞数为 $5 \times 10^{3}$ 个 $/ \mathrm{mL}$ 加于 96 孔培养板中, 每孔 100 $\mu \mathrm{L}$, 分样品组、对照组和空白组, 每组 4 个复孔. 于饱 和湿度, $5 \% \mathrm{CO}_{2}, 37{ }^{\circ} \mathrm{C}$ 条件下培养 $48 \mathrm{~h}$, 每孔加入 5 $\mathrm{mg} / \mathrm{mL}$ MTT 溶液 $10 \mu \mathrm{L}$, 继续培养 $4 \mathrm{~h}$ 后, 弃去上清液, 小心用 PBS 冲洗 3 遍, 然后每孔加入 DMSO $100 \mu \mathrm{L}$, 置 摇床上低速震荡 $10 \mathrm{~min}$, 使结晶充分溶解. 用酶联免疫 检测仪于 $570 \mathrm{~nm}$ 测量各孔的吸光值. 由公式: 抑制率 $=$ $\left(\mathrm{A}_{\text {对照 }}-\mathrm{A}_{\text {样品 }}\right) /\left(\mathrm{A}_{\text {对照 }}-\mathrm{A}_{\text {空白 }}\right) \times 100 \%$ 来计算不同浓度的抑 制率, 实验重复 3 次, 取平均值.

\section{References}

[1] Fang, X.-W.; Zhou, L.-Z.; Chen, Z.; Yang, M.-P.; Yang, B.-Q. Chin. J. Org. Chem. 2013, 33, 523 (in Chinese).

(方线文, 周丽珍, 成昭, 杨美盼, 杨秉勤, 有机化学, 2013, 33, 523.)

[2] Hyun, J.; Woo, Y.; Hwang, D.-S.; Jo, G.; Eom, S.; Lee, Y.; Park, J. C.; Lim, Y. Bioorg. Med. Chem. Lett. 2010, 20, 5510.

[3] Casano, G.; Dumetre, A.; Pannecouque, C.; Hutter, S.; Azas, N.;
Robin, M. Bioorg. Med. Chem. 2010, 18,6012

[4] Fang, F.; Huang, W.-D. J. Nucl. Agric. Sci. 2011, 25, 313 (in Chinese).

(方芳，黄卫东，核农学报, 2011, 25, 313.)

[5] Dong, X.-W. Ph.D. Dissertation, Zhejiang University, Hangzhou, 2009 (in Chinese).

(董晓武, 博士论文, 浙江大学, 杭州, 2009.)

[6] Yan, X.; Liu, H.-Q.; Zou, Y.-Q.; Ren, Z.-H. Chin. J. Org. Chem. 2008, 28, 1534 (in Chinese).

(延尔, 刘会青, 邹永青, 任占华, 有机化学, 2008, 28, 1534.)

[7] Xie, S.-Q.; Li, Q.; Zhang, Y.-H.; Wang, J.-H.; Mei, Z.-H.; Zhao, J.; Wang, C.-J. Apoptosis 2011, 16, 27.

[8] Wang, Y.-X.; Zhang, X.-B.; Zhao, J.; Xie, S.-Q.; Wang, C.-J. J. Med. Chem. 2012, 55, 3502.

[9] Zhao, J.; Liu, Y.; Zheng, M.-H.; Xie, S.-Q.; Wang, C.-J. Chin. J. Org. Chem. 2008, 28, 84 (in Chinese). (赵瑾，刘洋，郑梅花，谢松强，王超杰，有机化学, 2008, 28, 84.)

[10] Zheng, M.-H.; Gan, Y.; Xie, S.-Q.; Wang, C.-J. Zhao, J. Chin. J. Org. Chem. 2009, 29, 1445 (in Chinese).

(郑梅花，甘莹，谢松强，王超杰，赵瑾，有机化学，2009，29, 1445.)

[11] Chen, W.-M.; Guo, H.-X. Chin. J. Synth. Chem. 1999, 7, 422 (in Chinese). (陈万木, 郭宏雄, 合成化学, 1999, 7, 422.)

[12] Guan, L.-P.; Yin, X.-M.; Quan, H.-M.; Quan, Z.-S. Chin. J. Org. Chem. 2004, 24, 1274 (in Chinese). (关丽萍, 尹秀梅, 全红梅, 全哲山, 有机化学, 2004, 24, 1274.)

[13] Hu, Y.; Yang, Y.; Yu, Y.; Wen, G.; Shang, N.; Zhuang, W.; Lu, D.; Zhou, B.; Liang, B.; Yue, X.; Li, F.; Du, J.; Bu, X. J. Med. Chem. 2013, 56, 6033 .

[14] Mosmann, T. J. Immunol. Methods 1983, 65, 55. 